\title{
Inhaltsverzeichnis
}

Seite

\section{Zusammenfassender Bericht}

E. Kröner Die neuen Konzeptionen der Kontinuumsmechanik der festen Körper . . . . . . . . . . . . . . . . . . . . . 3

\section{Originalarbeiten}

E. MANN, R. v. JAN und A. SEEgER

Das elastische Verschiebungsfeld von Einzel- und Doppelkräften in anisotropen Medien . . . . . . . . . . . . . . 17

E. Gutsche Utber die elastischen Eigenschaften von Kristallgittern mit Wurtzitstruktur, Teil I . . . . . . . . . . . . . . . . 30

V. GEROLD Die Zonenbildung in Aluminium-Zink-Legierungen . . . .

W. LANge, A. Hässner und I. Berthold

Messung der Volumenselbstdiffusion in polykristallinem Zinn .

G. Gülzow-QUaL Ätzversuche an synthetischem Quarz . . . . . . . . . . . 62

H. KaRRAS Die Verfärbungsspektren von Strontiumfluorideinkristallen . . 68

S. OBERLÄNDER Anwendungsbeispiele der Prämissionsmethode auf Probleme der Reaktionskinetik in der Festkörperphysik . . . . . . . . 81

H. PAgnia Zum Aufbau dünner Kohlenwasserstoff-Polymerisatschichten mit verschiedener spezifischer elektrischer Leitfähigkeit . . . 90

\section{Kurze Originalmitteilungen}

Das Inhaltsverzeichnis dieses Teils befindet sich auf der letzten Seite des Heftes.

4. Vorabdruck der Titel und Abstracts von Originalarbeiten, die in den folgenden Heften dieser Zeitschrift oder in „Фицика Твердого Тела“ (Fizika Tverdogo Tela) erscheinen. 


\section{physica status solidi}

\section{Board of Editors}

P. AIGRAin, Paris, S. AMELINCKX, Mol-Donk, K. W. BÖER, New York, W. DEKEYSER, Gent, W. F R A N Z, Hamburg, P. G Ö R L I C H, Jena, E. G R I L L O T, Paris, R. K A I S C H E W, Sofia, P. T. L A N D SB E R G, Cardiff, L. NÉEL, Grenoble, A. P I E K A R A, Poznań, N. R I EHL, München, A. S E E G ER, Stuttgart, O. S T A S I W, Berlin, M. S T E E NB E C K, Jena, F. S T Ö C K M A NN, Karlsruhe, G. S Z I G E T I, Budapest, J. T A U C, Praha

$$
\text { Editor-in-Chief }
$$

K. W. BÖER, New York

$$
\text { Advisory Board }
$$

M. BALKANSKI, Paris, P. C. BANBURY, Reading, R. BERNARD, Paris, W. BRAUER, Berlin, W. COCH RAN, Cambridge, R. COELH O, Fontenay-aux-Roses, H.-D. DIETZE, Aachen, J. D. ESHELBY, Birmingham, H. K. HENISCH, Reading, G. J A COBS, Gent, J. JAUMAN N, Köln, E. KLIER, Praha, E. K ROENER, Cambridge Mass., M. MATYÁŠ, Praha, H.D. MEGAW, Cambridge,

T. S. MOSS, Camberley, E. NAGY, Budapest, E. A. NIE K IS CH, Erlangen,

L. PAL, Budapest, M. RODOT, Bellevue/Seine, B. V. ROLLIN, Oxford, H. M. ROSENBERG, Oxford, K.M. VAN VLIET, Minneapolis, R. VAUTIER, Bellevue/Seine

Volume 1 . Number 1 to 7

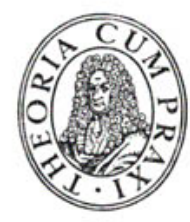

A K A D E M I E - V E R L A G - B E R L I N 
Subscriptions and orders for single copies should be addressed to

AKADEMIE-VERLAG GmbH, Berlin W 8, Leipziger Straße 3-4 or to Buchbandlung KUNST UND WISSEN, Erich Bieber, Stuttgart S, Wilhelmstr. 4-6 or to Deutsche Buch-Export und Import GmbH,

Leipzig C 1, Postschließfach 276

Schriftleiter und verantwortlich für den Inhalt: Professor Dr. K. W. Böer, New York 12, N. Y., 2 Washington Square Village. Redaktionskollegium: Dr. S. O berländer, Dr. E. Gut s ch e, W. B o r ch a rd t. Anschrift der Schriftleitung: Berlin C 2, Neue Schönhauser Str. 20, Fernruf: 422043 . Verlag: Akademie-Verlag GmbH, Berlin W 8, Leipziger Str. 3-4, Fernruf: 220441 , Telex-Nr. 011 773, Postscheckkonto: Berlin 35021 . - Die Zeitschrift "physica status solidi" erscheint monatlich; Bezugspreis eines Heftes DM 6,-. Bestellnummer dieses Bandes: 1068/1. Gesamtherstellung: VEB Druckerei "Thomas Müntzer" Bad Langensalza. - Veröffentlicht unter der Lizenznummer ZLN 5536 des Ministeriums für Kultur. Printed in Germany. 